\title{
Assessment of Adipose Tissue from Whole Body 3T MRI Scans
}

\author{
Ting Song ${ }^{1}$, Jing $A n^{2}$, Qun Chen², \\ Vivian Lee ${ }^{2}$, Andrew Laine ${ }^{1}$
}

${ }^{1}$ Department of Biomedical Engineering, Columbia University, New York, NY, USA ${ }^{2}$ Department of Radiology, New York University, New York, NY, USA

\section{IEEE EMBC 2005, Shanghai, China \\ September 3, 2005}




\section{Background}

- Adipose tissue quantification plays a central role in studying obesity in children and adults. Statistics show that $60 \%$ of American adults are either overweight or obese, and the rates are skyrocketing in children and adolescents.

- Using manual segmentation of MRI scanned images is a problem due to the high variation between manual delineations. Manual segmentation also requires highly trained experts with knowledge of anatomy.

- In this study, we used a specific water saturation sequence and histogram based segmentation method that provides robust delineation results for adipose tissue from whole body MRI scans. 


\section{Methodology}

- $\quad 3 T$ clinical T1-weighted MRI Acquisition

A standard T1-weighted sequence was used on a 3T whole body MR system for routine imaging. Data collected from this scan was compared to the water-saturation sequence described below.

- Water Saturation Acquisition for Fat

Since fat has a different Larmor frequency than water, a sufficiently narrow band RF (radio frequency) pulse can be used to tip water magnetization into the transverse plane and application of a spoiling gradient pulse can be used to dephase the magnetization. This combination is referred to as a "water saturation pulse." 


\section{Methodology}

Segmented Gradient Echo Acquisition

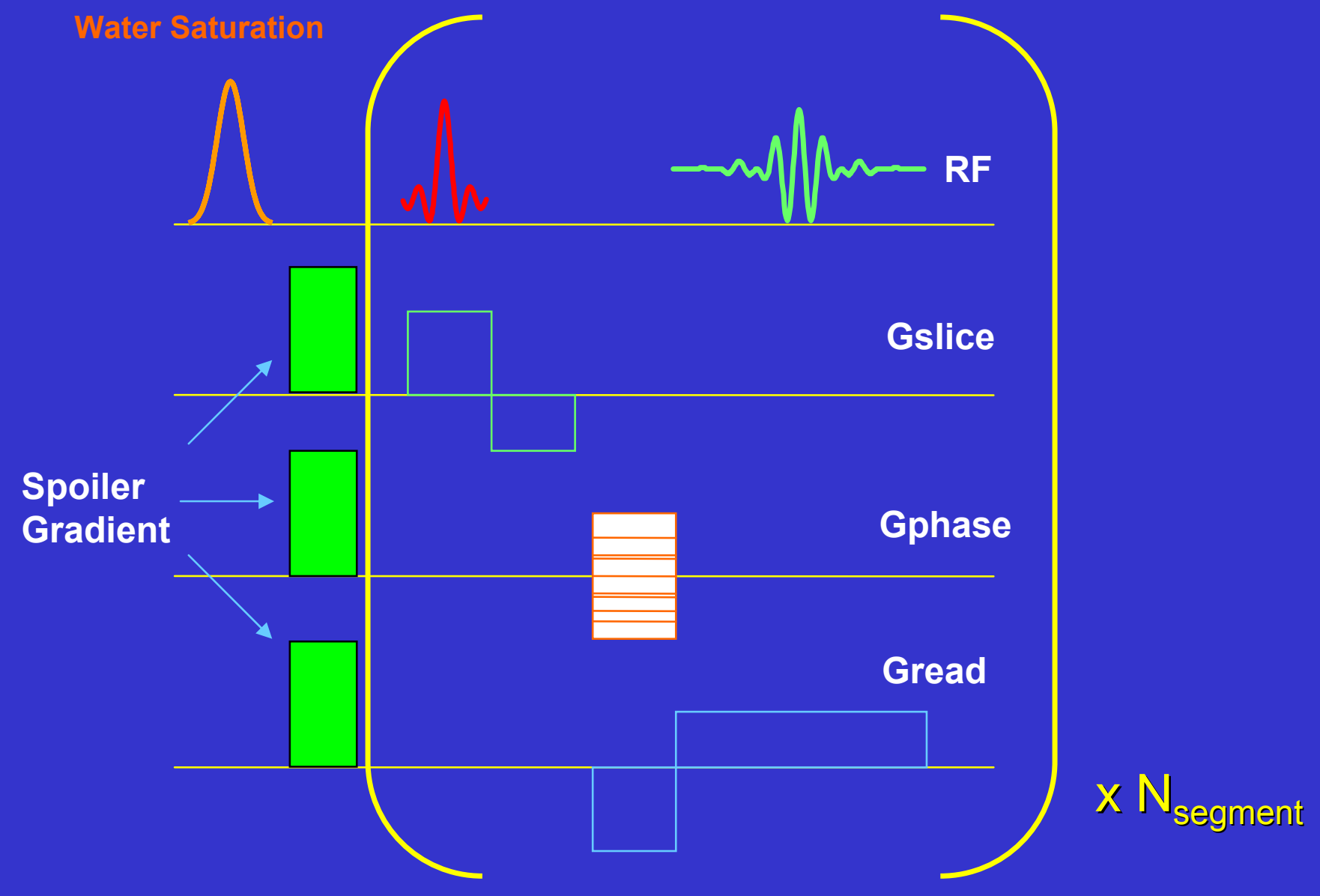

The total image acquisition time for each part is about 20 seconds. 


\section{Phantom Study}

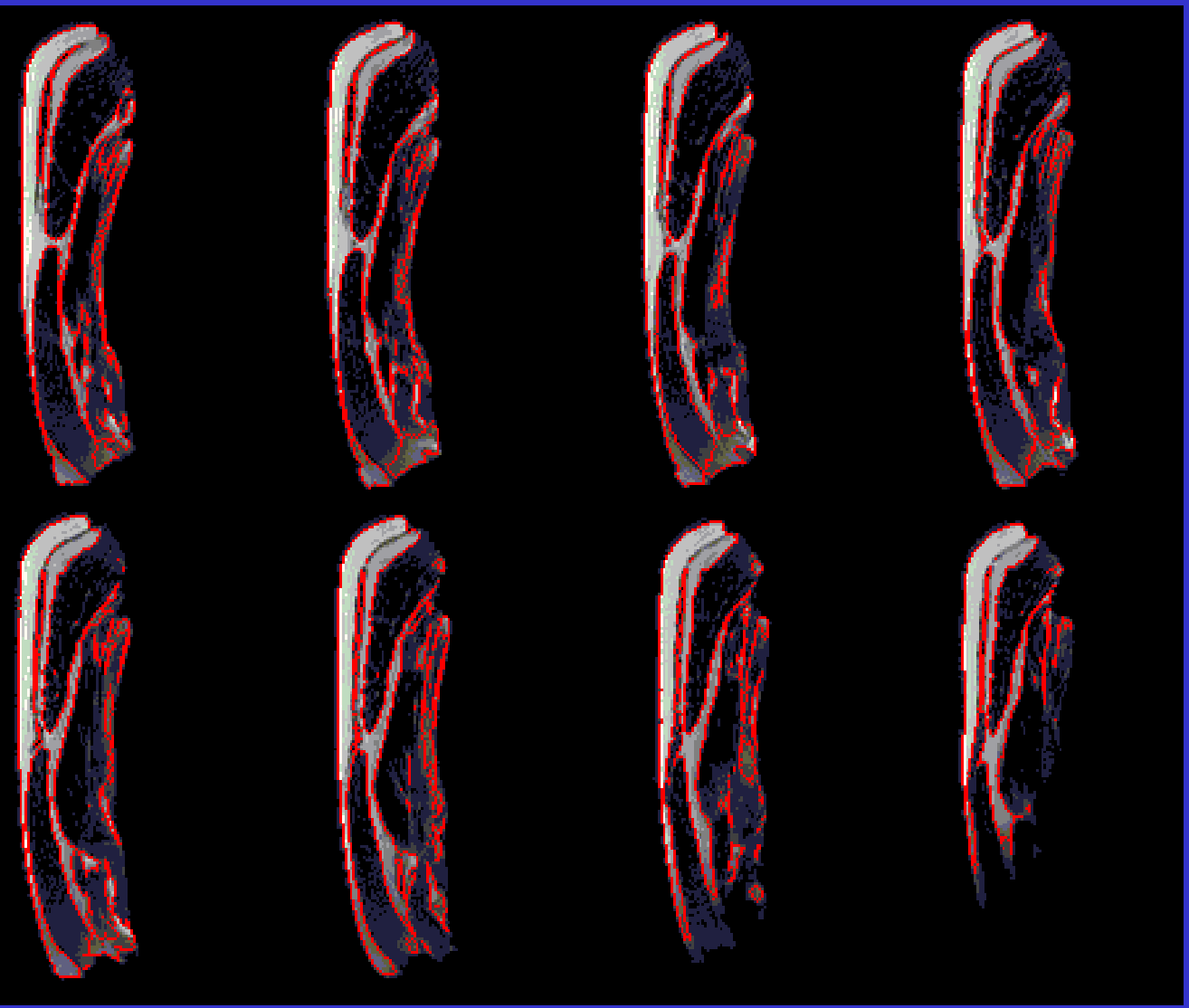

- We carried out a phantom study on fresh pig meat. Slice thickness is $5 \mathrm{~mm}$; pixel spacing is $1.46 \mathrm{~mm}$ by $1.46 \mathrm{~mm}$. Echo time (TE) is $1.64 \mathrm{~ms}$, and repetition time (TR) is $200 \mathrm{~ms}$. 


\section{Human Subjects Study}

- Four healthy volunteers (one female, three males) underwent 3T MR whole-body imaging for determination of fat distribution and quantification.

- MR examinations were performed on a 3T whole-body imager (Trio with TIM, Siemens); both regular clinical sequence and the water saturation sequence described above were applied. For the water saturation measurement, slice thickness was $10 \mathrm{~mm}$; pixel spacing was $1.95 \mathrm{~mm}$ by $1.95 \mathrm{~mm}$. Echo time (TE) was $2.32 \mathrm{~ms}$, and the repetition time (TR) was $280 \mathrm{~ms}$. 


\section{$\$$ Human Subjects Study: Water Saturation Method \\ and}

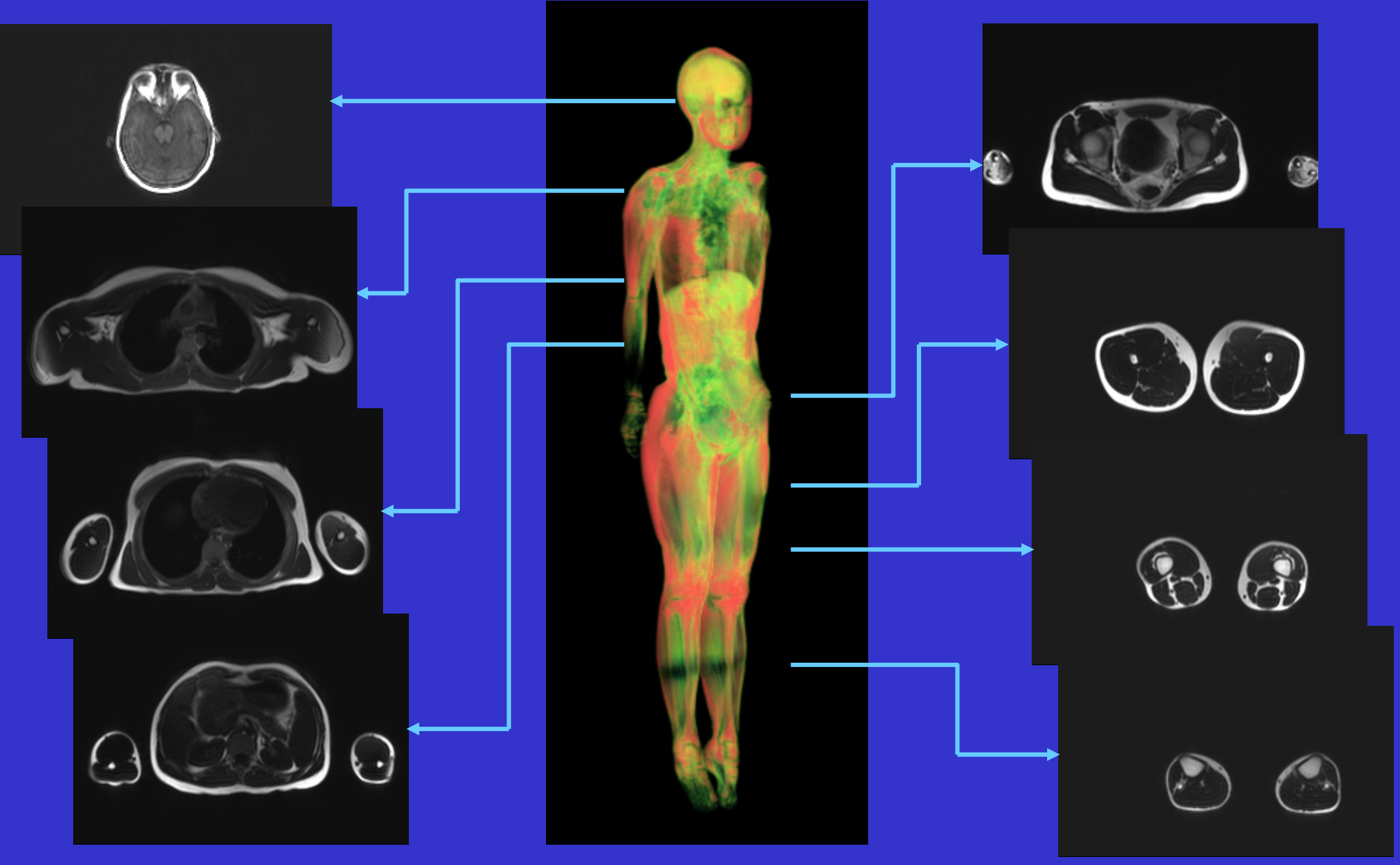

IEEE EMBC 2005, Shanghai, China 


\section{Preliminary Results}

Water Saturation Acquisition Method for Adipose Tissues
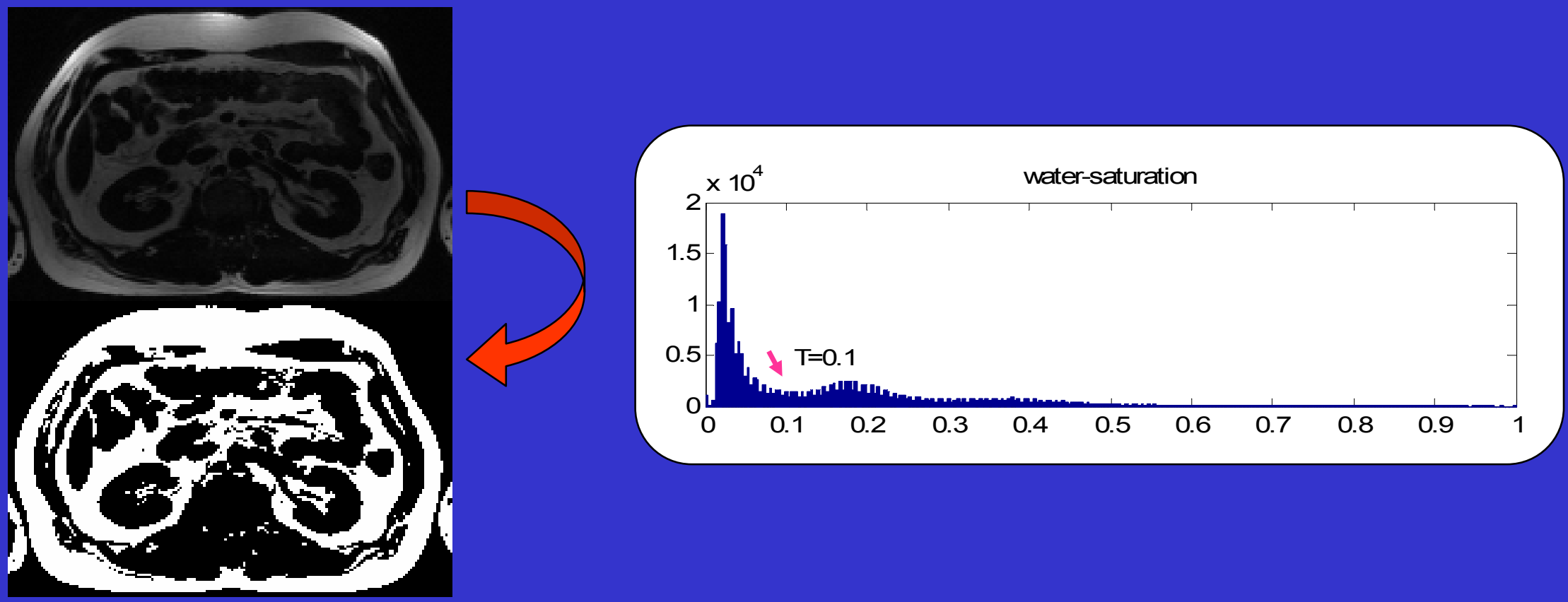

Our strategy for developing an adipose specific protocol was to reduce the complexity of the segmentation problem to the degree that simple methods could be applied successfully. We compared the results after histogram based threshold method on human subject's data. The valley in the water saturation histogram was a reasonable threshold value to separate fat. 


\section{Preliminary Results}

\section{Clinical T1 in-phase}
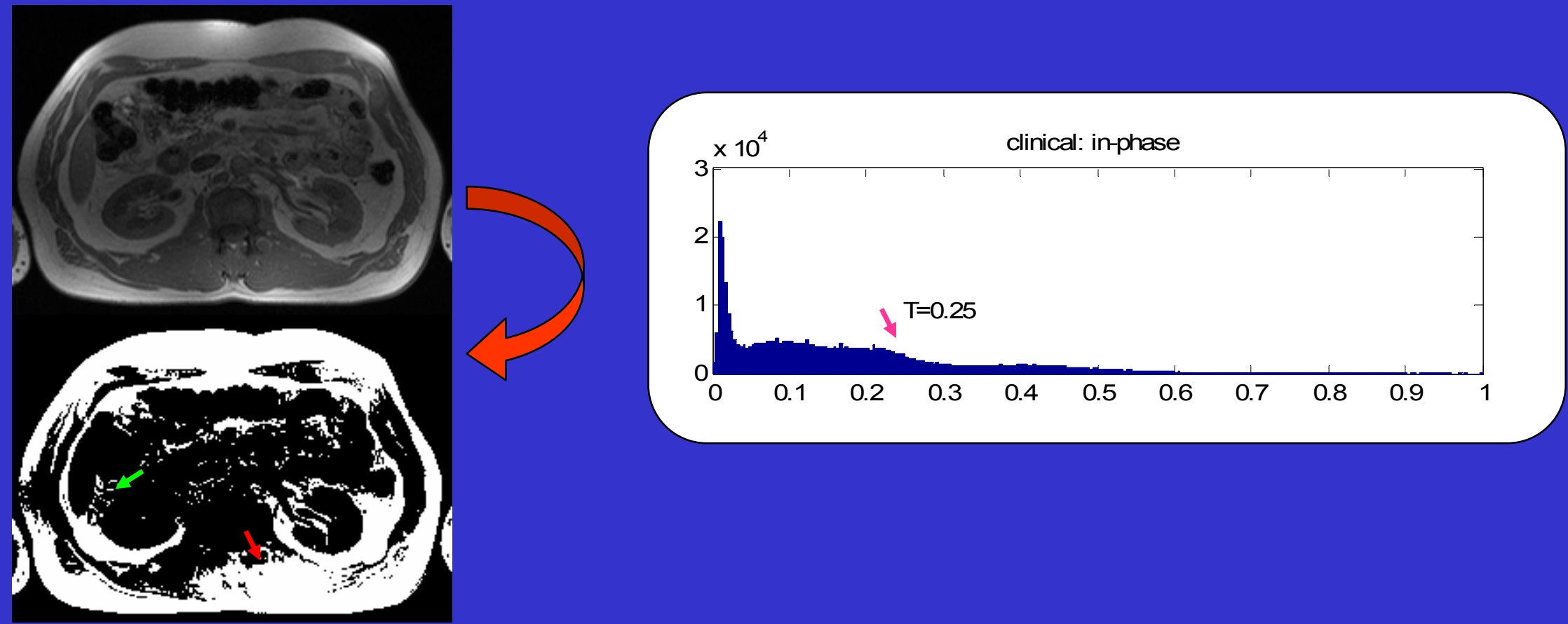

In comparison, clinical in-phase acquisition could not provide consistent intensity values within fat because over-segmentation and undersegmentation appeared when same threshold method was applied. Furthermore, there was no clear valley in the histogram to provide an effective threshold level. 


\section{Preliminary Results}

\section{Clinical T1 out-of-phase}
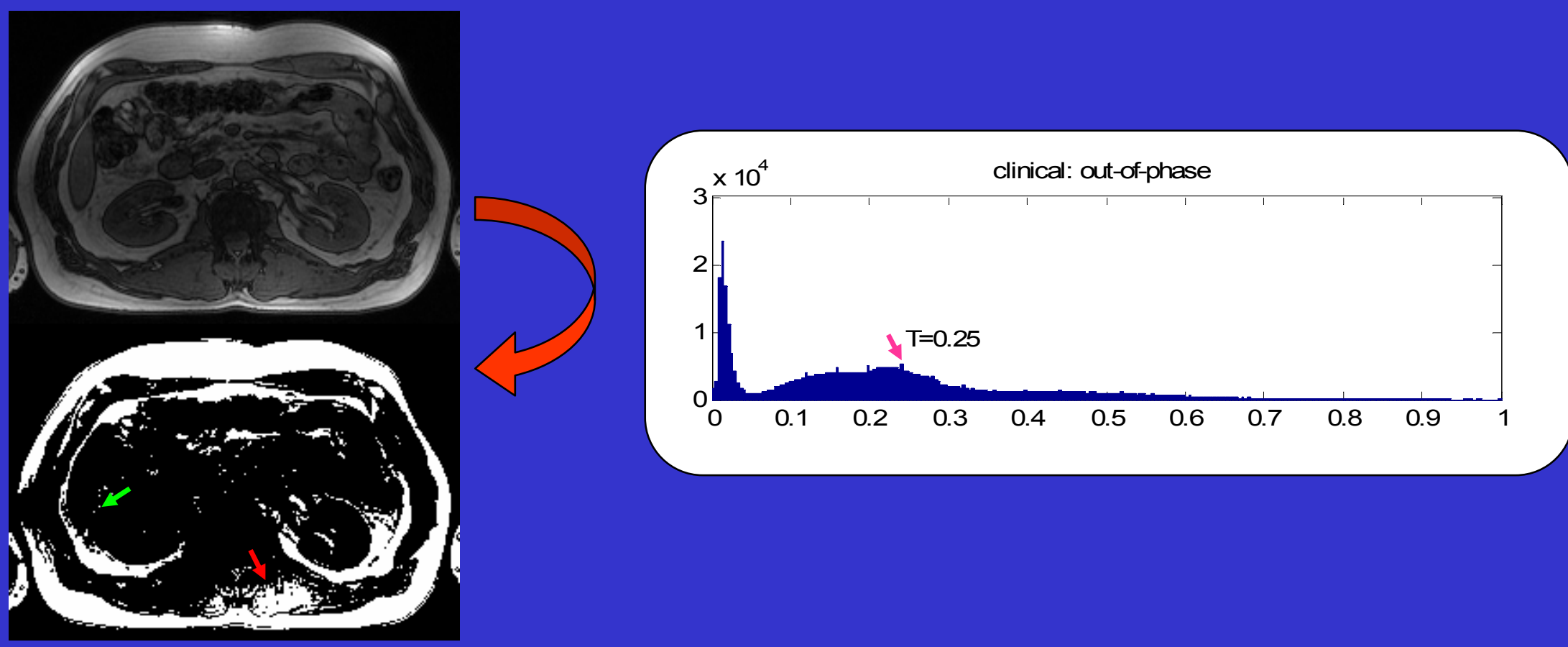

Similarly to clinical in-phase results, clinical out-of-phase acquisition could not provide consistent intensity values within fat because oversegmentation and under-segmentation appeared. 


\section{Future Research}

- In the future, we plan to focus on diseases correlated with adipose distribution such as Type II diabetes and liver diseases.

- Building a 3D adipose anatomical database as a resource for clinical studies will be longitudinal study, which would include screening many patients across age, race, and gender and creating a library of 3D adipose "skeletons." 


\section{Conclusions}

- The study of whole body fat distribution is becoming recognized as an important metric in the diagnostic screening of disease and therapy.

- Instead of manually segmenting fat from other tissues, an effective water saturation acquisition method and a histogram based segmentation method provided robust delineation of adipose tissue from whole body MRI scans.

- Both phantom and human subject studies were performed. Compared with a standard clinical 3T T1-weighted acquisition protocol, our method gave superior results and suggests a promising new high-speed protocol suitable for screening patients of all ages. 


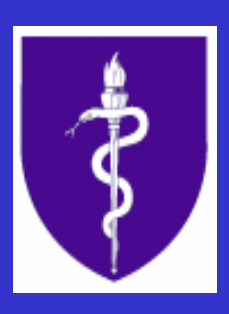

\section{Thank you !}

\section{http://hbil.bme.columbia.edu}

